\title{
Association of Alterations in Intestinal Microbiota With Impaired Psychological Function in Patients With Inflammatory Bowel Diseases in Remission
}

Humbel, Fabienne ; Rieder, Jessica Harrell ; Franc, Yannick ; Juillerat, Pascal ; Scharl, Michael ; Misselwitz, Benjamin ; Schreiner, Philipp ; Begré, Stefan ; Rogler, Gerhard ; von Känel, Roland ; Yilmaz, Bahtiyar ; Biedermann, Luc

\begin{abstract}
BACKGROUND AIMS Depression and anxiety are frequent comorbidities with inflammatory bowel diseases (IBD). Alterations to the intestinal microbiome promote not only intestinal inflammation but also psychologic function. We studied the interactions between the composition of the intestinal microbiota and psychological outcomes in patients with IBD in Switzerland. METHODS We performed a prospective study of psychological comorbidities and quality of life (QoL) in 171 participants in the Swiss IBD Cohort Study with IBD in remission. Participants complete the Hospital Anxiety and Depression Scale, Perceived Stress Questionnaire, the 36-Item Short Form Survey, and the IBD QoL Questionnaire. Microbes were collected from intestinal biopsies and analyzed by 16S rRNA high-throughput sequencing. RESULTS Microbiomes of patients with higher perceived stress had significantly lower alpha diversity. Anxiety and depressive symptoms were significantly associated with beta diversity. We found a negative correlation between psychological distress and abundance of Clostridia, Bacilli, Bacteroidia, and Betaand Gamma-proteobacteria. Psychological distress was also associated with decreases in operational taxonomic units from the lineages of Lachnospiraceae, Fusobacteriaceae, Ruminococcaceae, Veillonellaceae, Alcaligenaceae, Desulfovibrionaceae, and Bacteroidaceae families. The relative abundance of Bifidobacterium in patients with Crohn's disease and Desulfovibrio in patients with ulcerative colitis correlated with depression, whereas abundance of Sutterella, RF 32, and Lactococcus correlated with quality of life in patients with Crohn's disease. CONCLUSIONS We identified correlations between the composition of the intestinal microbiota in patients with IBD and remission, psychological well-being, and QoL. Further studies should investigate how intestinal inflammation, the microbiome, and microbial metabolites affect psychological well-being and whether these components are mono- or bi-directionally linked.
\end{abstract}

DOI: https://doi.org/10.1016/j.cgh.2019.09.022

Posted at the Zurich Open Repository and Archive, University of Zurich

ZORA URL: https://doi.org/10.5167/uzh-178714

Journal Article

Accepted Version

Originally published at:

Humbel, Fabienne; Rieder, Jessica Harrell; Franc, Yannick; Juillerat, Pascal; Scharl, Michael; Misselwitz, Benjamin; Schreiner, Philipp; Begré, Stefan; Rogler, Gerhard; von Känel, Roland; Yilmaz, Bahtiyar; Biedermann, Luc (2020). Association of Alterations in Intestinal Microbiota With Impaired Psychological 
Function in Patients With Inflammatory Bowel Diseases in Remission. Clinical Gastroenterology and Hepatology, 18(9):2019-2029.e11.

DOI: https://doi.org/10.1016/j.cgh.2019.09.022 


\title{
Association of Alterations in Mucosal Intestinal Microbiota with Impaired Psychological Function in Patients with Inflammatory Bowel Diseases in Remission
}

\author{
Short Title: Microbial \& Psychological Alterations in IBD
}

Fabienne Humbel $^{1,}{ }^{\ddagger}$, Jessica Harrell Rieder ${ }^{2,}{ }^{\ddagger}$, Yannick Franc $^{3}$, Pascal Juillerat $^{2,4}$, Michael Scharl $^{1}$, Benjamin Misselwitz ${ }^{1,2,4}$, Philipp Schreiner ${ }^{1}$, Stefan Begré5,6, Gerhard Rogler ${ }^{1}$, Roland von Känel , Bahtiyar Yilmaz ${ }^{2 *}$ and Luc Biedermann ${ }^{1^{*}}$ on behalf of the Swiss IBD Cohort Study Group ${ }^{\circ}$

${ }^{1}$ Department of Gastroenterology \& Hepatology, University Hospital Zurich, University of Zurich, Zurich, Switzerland.

${ }^{2}$ Maurice Müller Laboratories, Department for Biomedical Research, Inselspital, Univertsity of Bern, Switzerland

${ }^{3}$ Center for Primary Care and Public Health (Unisanté), University of Lausanne, Switzerland

${ }^{4}$ Gastroenterology, Clinic for Visceral Surgery and Medicine, Bern University Hospital, University of Bern, Bern, Switzerland.

${ }^{5}$ Neurology, Department of Biomedical Research, Bern University Hospital, University of Bern, Bern, Switzerland

${ }^{6}$ ISFOM - Institute of Stress Diseases and Stressmanagement, Weinbergstrasse 139, CH-8006 Zurich, Switzerland

${ }^{7}$ Department of Consultation-Liaison Psychiatry and Psychosomatic Medicine, University Hospital Zurich, University of Zurich, Zurich, Switzerland

${ }^{\ddagger}$ co-first authors, equal contributions

* co-last authors, equal contributions

Corresponding authors:

Luc Biedermann, Department of Gastroenterology \& Hepatology, University Hospital Zurich. Raemistrasse 100, 8091 Zurich, Switzerland. luc.biedermann@usz.ch. Phone +41442551111

Bahtiyar Yilmaz, Department for Biomedical Research, Inselspital, University of Bern, Murtenstrasse 35, 3008, Bern, Switzerland. Bahtiyar.yilmaz@dbmr.unibe.ch. Phone: +41 316328682

Conflict of interest: none of the authors reports any conflict of interest regarding this study.

Author Contribution

FH, LB, GR and RvK formed the concept of the study. SB, GR and RvK carefully selected psychological outcome parameters for SIBDCS patients' questionnaires. YF and BY performed pre-evaluations for data extraction from the SIBDCS. JHR, PJ, AJM and BY collected intestinal biopsy samples and coordinated microbial pre-analytics as well as bioinformatics. LB, YF and BY carried out first analyses of data. YF and BY performed the final statistical analysis. FH and LB drafted and FH, LB, BY wrote the manuscript. BM, MS, PS, PJ, JHR and GR gave critical input at all writing stages; all authors read and approved the final manuscript. 


\section{'Members of the SIBDCS study group:}

Karim Abdelrahman, Gentiana Ademi, Patrick Aepli, Amman Thomas, Claudia Anderegg, Anca-Teodora Antonino, Eva Archanioti, Eviano Arrigoni, Diana Bakker de Jong, Bruno Balsiger, Polat Bastürk, Peter Bauerfeind, Andrea Becocci, Stefan Begré, Dominique Belli, José M. Bengoa, Luc Biedermann, Janek Binek, Mirjam Blattmann, Stephan Boehm, Tujana Boldanova, Jan Borovicka, Christian P. Braegger, Stephan Brand, Lukas Brügger, Simon Brunner, Patrick Bühr, Bernard Burnand, Sabine Burk, Emanuel Burri, Sophie Buyse, Dahlia-Thao Cao, Ove Carstens, Dominique H. Criblez, Sophie Cunningham, Fabrizia D’Angelo, Philippe de Saussure, Lukas Degen, Joakim Delarive, Christopher Doerig, Barbara Dora, Susan Drerup, Mara Egger, Ali El-Wafa, Matthias Engelmann, Jessica Ezri, Christian Felley, Markus Fliegner, Nicolas Fournier, Montserrat Fraga, Yannick Franc, Pascal Frei, Remus Frei, Michael Fried, Florian Froehlich, Raoul Ivano Furlano, Luca Garzoni, Martin Geyer, Laurent Girard, Marc Girardin, Delphine Golay, Ignaz Good, Ulrike Graf Bigler, Beat Gysi, Johannes Haarer, Marcel Halama, Janine Haldemann, Pius Heer, Benjamin Heimgartner, Beat Helbling, Peter Hengstler, Denise Herzog, Cyrill Hess, Roxane Hessler, Klaas Heyland, Thomas Hinterleitner, Claudia Hirschi, Petr Hruz, Pascal Juillerat, Carolina Khalidde Bakker, Stephan Kayser, Céline Keller, (Christina Knellwolf (-Grieger)), Christoph Knoblauch, Henrik Köhler, Rebekka Koller, Claudia Krieger(-Grübel), Patrizia Künzler, Rachel Kusche, Frank Serge Lehmann, Andrew Macpherson, Michel H. Maillard, Michael Manz, Astrid Marot, Rémy Meier, Christa Meyenberger, Pamela Meyer, Pierre Michetti, Benjamin Misselwitz, Patrick Mosler, Christian Mottet, Christoph Müller, Beat Müllhaupt, Leilla Musso, Michaela Neagu, Cristina Nichita, Jan Niess, Andreas Nydegger, Nicole Obialo, Diana Ollo, Cassandra Oropesa, Ulrich Peter, Daniel Peternac, Laetitia Marie Petit, Valérie Pittet, Daniel Pohl, Marc Porzner, Claudia Preissler, Nadia Raschle, Ronald Rentsch, Alexandre Restellini, Sophie Restellini, Jean-Pierre Richterich, Frederic Ris, Branislav Risti, Marc Alain Ritz, Gerhard Rogler, Nina Röhrich, Jean-Benoît Rossel, Vanessa Rueger, Monica Rusticeanu, Markus Sagmeister, Gaby Saner, Bernhard Sauter, Mikael Sawatzki, Michael Scharl, Martin Schelling, Susanne Schibli, Hugo Schlauri, Dominique Schluckebier, Daniela Schmid, Sybille Schmid (-Uebelhart), Jean-François Schnegg, Alain Schoepfer, Vivianne Seematter, Frank Seibold, Mariam Seirafi, Gian-Marco Semadeni, Arne Senning, Christiane Sokollik, Joachim Sommer, Johannes Spalinger, Holger Spangenberger, Philippe Stadler, Peter Staub, Dominic Staudenmann, Volker Stenz, Michael Steuerwald, Alex Straumann, Bruno Strebel, Andreas Stulz, Michael Sulz, Aurora Tatu, Michela Tempia-Caliera, Joël Thorens, Kaspar Truninger, Radu Tutuian, Patrick Urfer, Stephan Vavricka, Francesco Viani, Jürg Vögtlin, Roland Von Känel, Dominique Vouillamoz, Rachel Vulliamy, Paul Wiesel, Reiner Wiest, Stefanie Wöhrle, Samuel Zamora, Silvan Zander, Tina Wylie, Jonas Zeitz, Dorothee Zimmermann. 


\section{WHAT YOU NEED TO KNOW}

Background and Context: Patients with inflammatory bowel diseases (IBD) frequently have impaired psychological function, including anxiety and depression. The composition of the intestinal microbiome affects psychological function. We analyzed intestinal microbiota, isolated from intestinal biopsies of patients in remission from IBD, and compared it with psychological comorbidities.

New Findings: We correlated features of the intestinal microbiota composition in patients with IBD in remission and their psychological well-being and quality of life. Taxa that were reduced in patients with anxiety, depression, or reduced quality of life produce short-chain fatty acids.

Limitations: It is not clear whether the observed alterations in the intestinal microbiome contribute to or are a result of IBD and psychologic dysfunction.

Impact: Increasing our understanding of alterations to the intestinal microbiota in patients with IBD and impaired psychological function could help identify mechanisms of pathogenesis or treatments. 


\section{ABSTRACT}

BACKGROUND \& AIMS: Depression and anxiety are frequent comorbidities with inflammatory bowel diseases (IBD). Alterations to the intestinal microbiome promote not only intestinal inflammation but also psychologic function. We studied the interactions between the composition of the intestinal microbiota and psychological outcomes in patients with IBD in Switzerland.

METHODS: We performed a prospective study of psychological comorbidities and quality of life (QoL) in 171 participants in the Swiss IBD Cohort Study with IBD in remission. Participants complete the Hospital Anxiety and Depression Scale, Perceived Stress Questionnaire, the 36-Item Short Form Survey, and the IBD QoL Questionnaire. Intestinal microbiota were collected from intestinal biopsies and analyzed by $16 \mathrm{~S}$ rRNA high-throughput sequencing.

RESULTS: Microbiomes of patients with higher perceived stress had significantly lower alpha diversity. Anxiety and depressive symptoms were significantly associated with beta diversity. We found a negative correlation between psychological distress and abundance of Clostridia, Bacilli, Bacteroidia, and Beta- and Gamma-proteobacteria. Psychological distress was also associated with decreases in operational taxonomic units from the lineages of Lachnospiraceae, Fusobacteriaceae, Ruminococcaceae, Veillonellaceae, Alcaligenaceae, Desulfovibrionaceae, and Bacteroidaceae families. The relative abundance of Bifidobacterium in patients with Crohn's disease and Desulfovibrio in patients with ulcerative colitis correlated with depression, whereas abundance of Sutterella, RF 32 , and Lactococcus correlated with quality of life in patients with Crohn's disease.

CONCLUSION: We identified correlations between the composition of the intestinal microbiota in patients with IBD and remission, psychological well-being, and QoL. Further studies should investigate how intestinal inflammation, the microbiome, and microbial metabolites affect psychological well-being and whether these components are mono- or bi-directionally linked.

Keywords: psychology, bacteria, microbe, gut-brain interactions 


\section{INTRODUCTION}

The importance of the intestinal microbiota latter regarding onset and disease course of inflammatory bowel disease (IBD) is increasingly acknowledged ${ }^{1}$ and bi-directional host-microbe interactions might either promote or reduce intestinal inflammation ${ }^{2}$. Previous studies on fecal bacterial composition identified a reduced level of representatives of Firmicutes and an increased level of representatives of Proteobacteria in Crohn's disease (CD) patients while in ulcerative colitis (UC) a lower extent of intestinal microbial alterations was found compared to $\mathrm{CD}^{3,4}$. However, whether these alterations are primary drivers of IBD (and if so to what extent) or secondary to the underlying inflammation has not been sufficiently clarified ${ }^{3}$.

Intestinal microbiota interacts with the central nervous system, therefore potentially modulating behavior, mood and cognitive function ${ }^{5}$. In this evolving concept of a gut-brain axis, microbiota constitutes an integral part of a complex bidirectional communication system via endocrine, metabolic and neural signals, with microbial metabolites affecting the intestinal immune system, submucosal autonomic nervous system ${ }^{6}$, hormonal signaling to the brain or the brain directly via bloodstream ${ }^{7}$.

The prevalence of clinically relevant depressive symptoms has consistently been reported to be increased in IBD patients, particularly during active disease ${ }^{8}$. In addition, increased levels of stress and anxiety are major drivers of IBD relapse and severity ${ }^{9,10}$. Previous studies have shown that patients with depression and other mood disturbances also show distinctive alterations in their intestinal microbial composition ${ }^{6,11}$. The first direct proof of a microbial-derived effect on cognition in humans was obtained in a randomized double-blind controlled trial of healthy women receiving either probiotics or placebo ${ }^{12}$. The activity of distinctive brain regions involved in the processing of emotion and sensation was significantly modified in women who had received the probiotic preparation. Until now, there are only few interventional studies in humans on the potential interplay of intestinal microbial composition and psychological distress $^{12-14}$

Perceived stress was shown to be associated with exacerbation of CD in in a prospective study from the Swiss IBD Cohort Study (SIBDCS). Interestingly, this association was fully derived by anxiety and depression components of perceived stres ${ }^{15}$. The increased risk of clinical recurrence with increased symptoms of anxiety and depression was confirmed in a more recent prospective investigation in over 2000 SIBDCS patients ${ }^{16}$. Quality of life (QoL) in IBD patients is impaired compared to healthy individuals, particularly in CD patients and during active disease ${ }^{17}$. Decrease in $Q o L$ may even indicate subclinical disease activity ${ }^{18}$ and is associated with an increase in risk of subsequent flares.

Taken together, a large body of evidence indicates a strong association of the microbiota with mood, stress, anxiety and depression. We therefore aimed to investigate the relationship of mucosa-associated microbial composition with psychological factors, namely perceived stress, anxiety and depression symptoms as well as QoL in patients with IBD in remission. 


\section{Methods}

\section{Swiss SIBDCS and Patient selection}

The SIBDCS is a nation-wide disease-oriented prospective cohort, including patients from all over Switzerland since 2006 including currently more than 3100 patients. An annual follow-up questionnaire is sent to the patients covering the clinical disease course, epidemiological data and validated questionnaires for anxiety, depression, perceived stress and quality of life (QoL) among others. All patients provided written informed consent to participate in the study ${ }^{19}$. Over a thousand samples from different intestinal regions from 376 patients were sequenced and of those patients, 204 had completed at least one patient questionnaire and contained a sufficient number of reads ( $\geq 4500)$. At inclusion, the patient's physician filled an enrollment questionnaire to make a clinical phenotyping of the disease activity (Crohn's disease activity index (CDAI) for CD and modified Truelove and Witt's activity index (MTWAI), for UC). Patients with $\mathrm{CDAl}<150$ or MTWAI 66 are in remission. We therefore excluded 33 patients with active disease at the time of biopsy due to three main reasons: first, prevalence of psychological symptoms including anxiety and depression is considerably affected by disease activity (and potentially vice versa); second, in active disease inflammation might more readily be driven by frank systemic inflammation rather than altered intestinal microbiota ${ }^{8}$; third, intestinal microbial composition differs in active disease vs. IBD in remission ${ }^{20,21}$. Hence, samples from 204 patients remained for further analysis.

\section{Scores for depression, anxiety, perceived stress and quality of life}

The Questionnaire of the SIBDCS includes validated scores to assess depression, anxiety, perceived stress (PSQ) and Quality of Life-36-Item Short Form Survey (SF-36). All items comprising these scores are exclusively being obtained within the patients' questionnaire, thus representing genuine patient reported outcomes. To investigate potential associations with bacterial composition, we used continuously scale measures and established cut-offs as described in the supplementary material, using the Hospital Anxiety and Depression Scale (HADS) for anxiety and depression (HADSA) and depression (HADS-D) ${ }^{22}$, the Perceived Stress Questionnaire (PSQ) ${ }^{23}$, the 36-item Short Form Health Survey (SF36), and the disease-specific Inflammatory Bowel Disease Questionnaire (IBDQ) for Quality of Life (QoL) ${ }^{24}$.

\section{Microbial profiling and statistical analyses}

The mucosa-associated microbiota of IBD patients was analyzed by $16 \mathrm{~S}$ rRNA amplicon sequencing on the lonTorrentPGM ${ }^{\mathrm{TM}}$ platform ${ }^{25}$ and detailed in the supplementary material. 


\section{RESULTS}

\section{Baseline characteristics of participants and 165 rRNA sequencing}

To characterize the microbial communities of IBD patients in the context of their psychological distress and quality of life, we included 171 IBD patients into this study (S-Table 1, 2; S-Results). We included comparable numbers of CD and UC patients with even gender distribution (Female: $56 \%$ ).

\section{Microbiota species richness (alpha-diversity) according to psychological distress and quality of life}

We first tested whether psychological distress scores were correlated with changes in species richness, as assessed by the Shannon and Simpson indices of alpha diversity. The Shannon index was reduced in IBD patients with an increased perceived stress score (PSQ $>0.45$ ) as compared to those with a lower perceived stress score $(P S Q<0.45)(S-F i g u r e ~ 1)$. This difference was only observed for the overall IBD population but did not reach significance in the subgroups of CD or UC patients.

We then analyzed overall species richness in CD and UC patients according to anxiety and depression (Figure 1). We noted a significant reduction in species richness with increasing anxiety in UC patients (Figure 1a) and with increasing depression in CD patients (Figure $1 b)$. In addition, there was a minor non-significant trend $(p<0.15)$ for lower species richness with increased anxiety in CD and depression in UC patients.

\section{Microbial compositional differences (beta-diversity) according to psychological distress}

We then tested for microbial differences in categorized disease groups according to psychological distress scores for IBD overall, CD and UC patients according to anxiety (Figure 2a) and depression (Figure 2b). We identified significant clustering in several groups according to the extent of anxiety and depression. Regarding anxiety, we identified significant differences between the groups with no or low (HADS-A<7) vs. moderate or high anxiety (HADS-A>8). This difference remained robust when different cut-off values for anxiety were used (subgroup-1: HADS-A>10 vs. HADS-A<8; subgroup-2: HADS-A>14 vs. HADS-A<8; Figure 2a). Similar results were observed for depression (Figure $2 b)$. Since the $p$ value for beta dispersion was lower 0.05 , we therefore cannot exclude that dispersion also contributes to the clustering in anxiety and depression (Figure 2). We also observed significant microbial clustering according to perceived stress with differences in the groups with $P S Q \geq 0.45$ vs. $P S Q<0.45$ in IBD overall (S-Figure 2), but not in the subgroups of $C D$ and $U C$ patients (not shown). Of note, we observed that samples from the same individual patient showed a considerable intraindividual clustering regardless of the site where the biopsies (from ileum to rectum) were obtained (S-Figure 3).

\section{Specific taxonomical differences in bacterial composition according to the extent of psychological distress}

Next, we compared the relative abundances across disease subtypes according to the extent of psychological distress ${ }^{3}$. A comprehensive summary of bacteria with a significant association with anxiety, depression, perceived stress and reduced QoL in CD or UC is provided in S-Table 3. At the phylum level, only Fusobacterium was significantly reduced with increasing anxiety. Most of the taxonomic changes at order, family and genus level were observed within the phylum Firmicutes, with lesser changes also within the phyla Bacteroidetes, Actinobacteria or Proteobacteria. 
The microbial profile in CD and UC patients also correlated to the depression state (Figure 3 and S-Table 3). As described for anxiety, most taxonomic changes were observed within the Firmicutes phylum. Further the relative abundance of S24-7, Eubacterium, Clostridiales, Lachnospiraceae, Roseburia, Ruminococcus was negatively correlated with depression in CD (Figure 3a), while Erysipelotrichaceae Lachnospira, Blautia, Phascolarctobacterium, Streptococcus showed a similar negative correlation in UC (Figure 3b). Positive correlations with depression were observed between Bifidobacterium in CD and Desulfovibrio in UC (Figure 3 and S-Table 3). Overall, we demonstrate several negative correlations between depression and relative abundance of several taxa within the Firmicutes phylum whose roles need to be further investigated.

Performing a similar correlation analysis for anxiety (Figure 4 and S-Table 3), we only observed significant associations with abundance of taxa were in CD but not in UC. Most of the taxonomic changes were observed for Firmicutes, Bacteroidetes and Proteobacteria and to a lesser extent for Actinobacteria phyla and abundance was generally reduced with increasing anxiety (Figure 4 and S-Table 3).

QoL correlated with abundance of taxa in CD and UC and significant associations were observed for 6 taxa for each condition (Figure 5 and S-Table 3). The relative abundance of the RF32 family, Sutterella and Lactococcus correlated positively with QoL. Some of the remaining implicated taxa including Phascolarctobacterium, Streptococcus and Blautia had also been identified in our correlation analyses for depression or anxiety (Figure 3, 4, 5 and S-Table 3). However, associated taxa for both outcomes only partially overlapped and no clear pattern was discernible (Figure 3, 4, 5 and STable 3).

Perceived stress correlated negatively with Sutterella, Haemophilus, Lachnospira, Parabacteroides, RF32 family and Eubacterium abundance in UC patients (Figure 5c and S-Table 3). A similar negative association of those taxa had been observed with depression (Figure 4). Of note, Sutterella and RF32 family abundancy increased with higher QoL (Figure 5b), pointing to these taxa as markers for favorable psychological outcomes. No significant associations of microbial profiles and perceived stress were observed in CD.

Using the extended portfolio of taxa associated with psychological distresses in our dataset, we used machine learning aiming to identify bacterial lineages that best correlated with adverse vs. normal psychological functioning (STable 4, 5, 6). 


\section{DISCUSSION}

We demonstrate an association of psychological outcome parameters including anxiety, depression and perceived stress with differences in mucosa-associated microbiota diversity as well as distinctive alterations in microbiota abundancy in IBD patients in remission. After predominantly descriptive initial investigations on microbial differences between IBD patients and healthy controls and an ongoing debate on whether these alterations implicate rather cause or effect, an increasing body of recent evidence points to the intestinal microbiota as an active player in the pathogenesis of $\mathrm{IBD}^{26,27}$. However, the role of the microbiota is less clear in gut-brain axis. For both, animal and human studies, the majority of investigations largely remain scratching on the descriptive or associative level and interventional studies remain scarce. Further, in most studies the microbiota was assessed by the proxy fecal microbial composition from stool samples which differs from the mucosa-associated microbiota, relevant for the bacteria-host interaction ${ }^{28}$.

In this study we found differences in the composition of the mucosa-associated microbiota in IBD patients with vs. without psychological alterations. Of note, we observed these differences in a population of IBD patients with remission. This selection is crucial for two main reasons. First, changes in bowel movements per day were even in a nonIBD population shown to be associated with considerable alterations in intestinal microbiota composition ${ }^{29}$. Moreover, disease activity in patients with IBD in itself may have a substantial impact on intestinal microbial composition ${ }^{21}$, including relevant segmental differences in inflamed vs. non-inflamed tissue ${ }^{30}$. Second, psychological well-being, including anxiety, depression, PS and overall QoL may vary considerably with increases in disease activity, with even a reciprocal interaction, in that stress, anxiety and depressive symptoms may increase the risk of subsequent flare and vice versa ${ }^{16,31}$

Mechanistic aspects of interplay of psychiatric symptoms with microbial composition remains to be elucidated in future mechanistic investigation, including for instance on microbial metabolites. However, most of the $50^{\prime} 000$ peaks present in a typical mass spectrometry run have not even been characterized ${ }^{32}$, illustrating our lack of understanding of microbiota metabolism. A significant fraction of microbiota-derived metabolites will be absorbed in the intestine and distributed in the organism. In fact, in germ-free mice, colonization with a single $E$. coli species had an impact on the permeability of the intestinal barrier, suggesting that intestinal barrier is actively regulated ${ }^{33}$. Microbiota-derived metabolites regulate digestion and changes in the composition of the microbiome might alter the quantities and effects of these metabolites. Further, microbiota or microbiota-associated subclinical inflammation might affect the epithelial barrier and thus alter the influx of metabolites.

Our study has several strengths and limitations. The highly standardized and prospective acquisition of physician- and patient-derived data in the well-established SIBDC setting represents a strength of our study. Further, patients were selected for IBD in remission to minimize the risk of confounding IBD activity regarding microbial composition and psychological well-being. However, we acknowledge, that data on endoscopy to verify, that patients in clinical remission also are in endoscopic remission (which in UC is highly likely in the absence of rectal bleeding and normal stool frequency) is limited in our study and in SIBDCS - being designed at a time, where the importance of endoscopic outcome parameters was considerably less recognized - in general ${ }^{34}$.

Moreover, due to the detailed and extensive physician and especially also patient questionnaires in the SIBDCS our study could take a plethora of validated and well-established outcome variables into account. The longitudinal and standardized patient follow-up within SIBDCS including annual visits may be of particular relevance with regards to investigating psychosocial distress in IBD. Further, SIBDC is a long-term cohort study with a large number of 
questionnaires. Therefore, the risk of a Hawthorne effect, i.e. favorable patient responses regarding a single outcome in a study with a distinctive investigational aim, would be smaller which seems highly relevant for questions regarding psychological well-being ${ }^{35}$. In addition, we consider our mode of analysis of intestinal microbial composition using biopsy specimen and thus analyzing the mucosa-associated microbiota a strength as compared to the common use of fecal samples, only reflecting a proxy.

Limitations of our study include the cross-sectional and non-interventional design which does not allow to draw any firm conclusions on cause and effect. Furthermore, we are not able to make any comparison with non-IBD subjects. Evidently, the association between intestinal microbial and psychological alterations might also be reciprocal in that, changes in mood might subsequently modify gastrointestinal motility and behavior, including factors with a known effect on intestinal microbiota composition, such as intestinal motility/stool frequency ${ }^{29}$, diet ${ }^{36}$, or smoking ${ }^{37}$. We also cannot completely rule out, that microbiota-based identification and prediction of adverse psychological functioning reflects some degree of interference with (undetected) disease activity or exposure to other modifying factors, such as medical therapy that was not covered in the SIBDCS follow-up. This may specifically be the case with steroids, which not seldom are initiated by patients themselves on short-term. However, a recent study showed that corticosteroid do not have a strong influence on microbiota profile of Swiss IBD patient. Moreover, microbial composition also was not associated with the response to corticosteroids, as opposed for instance to anti-TNF therapy ${ }^{4}$. In addition, the assessment of psychiatric symptoms by questionnaires cannot replace a gold standard psychiatric interview. However, expert psychologists and psychiatrists were involved in the questionnaire design at the inception of the SIBDCS. Even though we used established and validated scores to obtain the psychological endpoint data important uncertainties remain. For example, different cultural background was not taken into account as some assessment instruments like perceived stress questionnaire are not entirely validated for trans-culturalism. Though we aimed for the shortest possible time interval in our study, the HADS assesses depression and anxiety in the past week only and the time interval between endoscopy and the questionnaire was $\leq 1$ month for 55 patients and $\leq 3$ months for 94 patients.

In conclusion, we identified that there are distinct taxonomic alterations in microbial composition in patients with IBD in relation to the extent of psychological distress, warranting further investigations. This includes changes in the abundance of distinctive microbial represents in correlation to the severity of anxiety, depression, perceived stress and impairment of QoL. Our study does not allow to gain further insights on cause and effect, pathogenetic mechanisms and the direction underlying these impairments in psychological well-being associated to microbial alterations. Nevertheless, our results imply, that the composition of the intestinal microbiota may not only be a crucial factor in the emergence of psychological distress but also a target of future microbiota-modulating therapeutic approaches aiming at modifying both course of IBD and associated psychological morbidity. 


\section{REFERENCES}

1. Miyoshi J, Chang EB. The gut microbiota and inflammatory bowel diseases. Transl Res. 2017;179:38-48. Epub 2016/06/14.

2. Macpherson AJ, Yilmaz B, Limenitakis JP, et al. IgA Function in Relation to the Intestinal Microbiota. Annu Rev Immunol. 2018;36:359-81.

3. Morgan XC, Tickle TL, Sokol H, et al. Dysfunction of the intestinal microbiome in inflammatory bowel disease and treatment. Genome Biol. 2012;13(9):R79.

4. Yilmaz BJ, P.; Oyas, O.; Ramon, C.; Bravo, F.; Franc, Y.; Fournier, N.; Geuking, M.; Michetti, P.; Mueller, C.; Pitter, V.; Maillard, M.; Rogler, G.; Wiest, R.; Stelling, J.; Macpherson, A.J. Microbial network disturbances in relapsing refractory Crohn's disease. Nature medicine. 2018.

5. Cryan JF, Dinan TG. Mind-altering microorganisms: the impact of the gut microbiota on brain and behaviour. Nat Rev Neurosci. 2012;13(10):701-12. Epub 2012/09/12.

6. Yarandi SS, Peterson DA, Treisman GJ, et al. Modulatory Effects of Gut Microbiota on the Central Nervous System: How Gut Could Play a Role in Neuropsychiatric Health and Diseases. J Neurogastroenterol Motil. 2016;22(2):201-12.

7. Petra Al, Panagiotidou S, Hatziagelaki E, et al. Gut-Microbiota-Brain Axis and Its Effect on Neuropsychiatric Disorders With Suspected Immune Dysregulation. Clin Ther. 2015;37(5):984-95.

8. Mikocka-Walus A, Knowles SR, Keefer L, et al. Controversies Revisited: A Systematic Review of the Comorbidity of Depression and Anxiety with Inflammatory Bowel Diseases. Inflamm Bowel Dis. 2016;22(3):75262.

9. Camara RJ, Ziegler R, Begre S, et al. The role of psychological stress in inflammatory bowel disease: quality assessment of methods of 18 prospective studies and suggestions for future research. Digestion. 2009;80(2):129-39.

10. Gracie DJ, Guthrie EA, Hamlin PJ, et al. Bi-directionality of Brain-Gut Interactions in Patients With Inflammatory Bowel Disease. Gastroenterology. 2018;154(6):1635-46.e3. Epub 2018/01/31.

11. Naseribafrouei A, Hestad K, Avershina E, et al. Correlation between the human fecal microbiota and depression. Neurogastroenterol Motil. 2014;26(8):1155-62. Epub 2014/06/01.

12. Tillisch K, Labus J, Kilpatrick L, et al. Consumption of fermented milk product with probiotic modulates brain activity. Gastroenterology. 2013;144(7):1394-401, 401 e1-4. Epub 2013/03/12.

13. Jiang $\mathrm{H}$, Ling $\mathrm{Z}$, Zhang $\mathrm{Y}$, et al. Altered fecal microbiota composition in patients with major depressive disorder. Brain Behav Immun. 2015;48:186-94. Epub 2015/04/13.

14. Steenbergen L, Sellaro R, van Hemert S, et al. A randomized controlled trial to test the effect of multispecies probiotics on cognitive reactivity to sad mood. Brain Behav Immun. 2015;48:258-64. Epub 2015/04/12.

15. Cámara RJ, Schoepfer AM, Pittet V, et al. Mood and nonmood components of perceived stress and exacerbation of Crohn's disease. Inflamm Bowel Dis. 2011;17(11):2358-65. Epub 2011/02/01.

16. Mikocka-Walus A, Pittet V, Rossel JB, et al. Symptoms of Depression and Anxiety Are Independently Associated With Clinical Recurrence of Inflammatory Bowel Disease. Clin Gastroenterol Hepatol. 2016;14(6):829-35.e1. Epub 2016/01/25.

17. Knowles SR, Graff LA, Wilding H, et al. Quality of Life in Inflammatory Bowel Disease: A Systematic Review and Meta-analyses-Part I. Inflamm Bowel Dis. 2018;24(4):742-51.

18. Cámara RJ, Juillerat $P$, Pittet $V$, et al. Quality of life: a potentially useful measure to indicate subclinical flares in Crohn disease. Intern Med J. 2012;42(7):e145-51.

19. Pittet V, Juillerat $P$, Mottet $C$, et al. Cohort profile: the Swiss Inflammatory Bowel Disease Cohort Study (SIBDCS). Int J Epidemiol. 2009;38(4):922-31. Epub 2008/09/09.

20. Neurath MF. Cytokines in inflammatory bowel disease. Nat Rev Immunol. 2014;14(5):329-42. Epub 2014/04/22.

21. Prosberg $\mathrm{M}$, Bendtsen $\mathrm{F}$, Vind $\mathrm{I}$, et al. The association between the gut microbiota and the inflammatory bowel disease activity: a systematic review and meta-analysis. Scand J Gastroenterol. 2016;51(12):1407-15. Epub 2016/08/09.

22. Snaith RP. The Hospital Anxiety And Depression Scale. Health Qual Life Outcomes. 2003;1:29. Epub 2003/08/01.

23. Levenstein S, Prantera C, Varvo V, et al. Development of the Perceived Stress Questionnaire: a new tool for psychosomatic research. J Psychosom Res. 1993;37(1):19-32.

24. Ware JE, Sherbourne CD. The MOS 36-item short-form health survey (SF-36). I. Conceptual framework and item selection. Med Care. 1992;30(6):473-83.

25. Yilmaz B, Spalinger MR, Biedermann L, et al. The presence of genetic risk variants within PTPN2 and PTPN22 is associated with intestinal microbiota alterations in Swiss IBD cohort patients. PloS one.

2018;13(7):e0199664. Epub 2018/07/03. 
26. Rehman A, Sina C, Gavrilova O, et al. Nod2 is essential for temporal development of intestinal microbial communities. Gut. 2011;60(10):1354-62.

27. Imhann F, Vich Vila A, Bonder MJ, et al. Interplay of host genetics and gut microbiota underlying the onset and clinical presentation of inflammatory bowel disease. Gut. 2018;67(1):108-19. Epub 2016/10/08.

28. Swidsinski A, Ladhoff A, Pernthaler A, et al. Mucosal flora in inflammatory bowel disease.

Gastroenterology. 2002;122(1):44-54.

29. Falony G, Joossens M, Vieira-Silva S, et al. Population-level analysis of gut microbiome variation. Science. 2016;352(6285):560-4.

30. Walker AW, Sanderson JD, Churcher C, et al. High-throughput clone library analysis of the mucosaassociated microbiota reveals dysbiosis and differences between inflamed and non-inflamed regions of the intestine in inflammatory bowel disease. BMC Microbiol. 2011;11:7.

31. Levenstein S, Prantera C, Varvo V, et al. Stress and exacerbation in ulcerative colitis: a prospective study of patients enrolled in remission. Am J Gastroenterol. 2000;95(5):1213-20.

32. Zampieri M, Sekar K, Zamboni N, et al. Frontiers of high-throughput metabolomics. Curr Opin Chem Biol. 2017;36:15-23. Epub 2017/01/05.

33. Uchimura Y, Fuhrer T, Li H, et al. Antibodies Set Boundaries Limiting Microbial Metabolite Penetration and the Resultant Mammalian Host Response. Immunity. 2018;49(3):545-59 e5.

34. Narula N, Alshahrani AA, Yuan Y, et al. Patient-Reported Outcomes and Endoscopic Appearance of Ulcerative Colitis: A Systematic Review and Meta-analysis. Clin Gastroenterol Hepatol. 2019;17(3):411-8 e3. Epub 2018/06/19.

35. Harrell CR, Gladwin BD, Hoag MP. Mitigating the "Hawthorne effect" in simulation studies.2722-9. Epub 2013.

36. David LA, Maurice CF, Carmody RN, et al. Diet rapidly and reproducibly alters the human gut microbiome. Nature. 2014;505(7484):559-63.

37. Biedermann L, Zeitz J, Mwinyi J, et al. Smoking cessation induces profound changes in the composition of the intestinal microbiota in humans. PLoS One. 2013;8(3):e59260. Epub 2013/03/14. 
Figure 1. Species richness changes with anxiety and depression. Correlation between alpha diversity and anxiety a) / depression b) in CD (red) and UC (blue) patients. Shannon and Simpson index (left and right panel) are plotted against anxiety values (HADS-D score) with regression lines. A LOESS smoothed fit curve demonstrating the confidence region is depicted in gray.

Figure 2. Microbial compositional differences associated with anxiety or depression. Beta diversity was calculated for a) anxiety and b) depression in all patients (overall: anxiety/ depression score $<7$ vs. $>8$ ), subgroup-1 (score $<8$ vs. $>10$ ) or subgroup-2 (score $<8$ vs. $>14$ ) for IBD overall and CD or UC patients. Categories with higher scores are shown in red, categories with lower score are shown in blue. Ellipses represent dispersion of samples with a $95 \%$ confidence interval surrounding each disease group.

Figure 3. Specific relative microbial abundances in IBD patients according to depression. Correlation of microbial taxa with depression in a) CD and b) UC patients are shown. Data were analyzed using MaAsLin with BH correction (q value) and only significant taxa $(q<0.05)$ are plotted. Red lines show significant positive correlations and green lines show significant negative correlations of relative abundance (arcsin-sqrt transformation) of identified taxa with depression scores (HADS-D).

Figure 4. Specific relative microbial abundances according to anxiety in CD. Correlations of microbial taxa with anxiety in CD patients are shown (in analogy to Figure 3).

Figure 5. Specific relative microbial abundances in association with quality of life and perceived stress. Correlations of microbial taxa with SF-36 values in a) CD patients and b) UC patients, and with PSQ scores in UC patients c) (similar to Figure 3). 